\title{
Matrix-Weighted Consensus with Leader-Following Topologies
}

\author{
Minh Hoang Trinh*, Mengbin Ye ${ }^{\dagger}$, Hyo-Sung Ahn*, and Brian D. O. Anderson ${ }^{\dagger}, \ddagger$
}

\begin{abstract}
In this paper, we report some results on a matrixweight consensus algorithm. Specifically, the leader-following graph is studied in detail. We study conditions for reaching a consensus in two types of leader-following graphs using the matrix-weighted consensus algorithm. The results will be used to interpret several social behaviors. Simulation results are also provided to vindicate the mathematical analysis.
\end{abstract}

\section{INTRODUCTION}

The consensus problem is a study of how multiple agents interact and exchange information in order to reach an agreement on one or more variables of interest. The problem of reaching a consensus has received sustained research attention from different communities over many years. In the control systems society, the consensus algorithm has received significant attention in the past 2 decades thanks to its ubiquitous applications in cooperative coordination of multi-agent systems [1]-[3]. Consensus may be applied to formation control of multiple autonomous vehicles [4], network synchronization [5], or distributed coordination of power networks [6], etc. In sociology, the consensus process [2], [7] has been closely linked to opinion dynamics [8][11]. Here, the variable of interest is the opinion value, on a given topic/issue, for individuals in a social network.

The usual consensus protocol successfully explained the process of reaching a consensus in one variable of interest, or on multiple unrelated variables of interest via the use of Kronecker products [2], [7], [10]. A recent extension has been to consider matrix-weighted graphs [12] to model the information flow. In short, a matrix-weighted graph is a graph with matrix weights, as compared to scalar weights, associated with each edge. The matrices are all square, with the same dimension, and each vertex is assumed to be storing a vector, rather than a scalar variable, of dimension consistent with the matrix dimension. Using matrix-weighted graphs, the authors in [12] introduced a matrix-weighted consensus algorithm and provided a necessary and sufficient condition for reaching a consensus with undirected graphs.

Several reasons motivate matrix-weighted consensus algorithms, and we briefly introduce them here. Consider firstly that exchanges of opinions between people are often complicated, especially if multiple related topics are being

\footnotetext{
*School of Mechanical Engineering, Gwangju Institute of Science and Technology (GIST), Gwangju, Republic of Korea. E-mail: \{trinhhoangminh, hyosung\}@gist.ac.kr

${ }^{\dagger}$ Research School of Engineering, The Australian National University, Canberra ACT 2601, Australia. Email: \{Mengbin.Ye, Brian. Anderson\} @anu.edu.au

${ }^{\ddagger}$ Data61-CSIRO (formerly NICTA), Canberra ACT 2601, Australia, and School of Automation, Hangzhou Dianzi University, Hangzhou 310018, China
}

discussed. Matrix weights are used to describe the interdependency of the related topics [13], [14]. In addition to this interdependency, matrix weights can also be used to describe topic-dependent interactions; depending on the topic being discussed, an individual may be experts or have only limited knowledge. The matrix weights then reflect how others in the social network perceive this individual on different topics.

Besides opinion dynamics on social networks, other problems involving coordination of multiple autonomous agents have used matrix-weighted consensus algorithms. Existing works include synchronizing coupled-oscillator networks [5], [15], bearing-based formation control and network localization [16], [17]. However, these require specific forms for the matrix weight, relevant only to the given problem. For example, the matrix weights in [16], [17] are orthogonal projection matrices obtained from bearing measurements. In this paper, we provide a result for a general class of matrix weights.

In the literature, leader-following interaction topologies have been well-studied in the context of the usual scalarweighted consensus algorithm [18], [19]. The authors in [20] studied leader-following graphs with communication delays. Consensus under fixed and switching leader-following graphs was investigated in [18], [21]. A treatment on consensus and clustering behaviors in leaderless and leader-following graphs for agents with double-integrator dynamics was provided in [19]. In this paper, we study matrix-weighted consensus algorithm with leader-following interaction topologies. The leader-following graphs may be used to model how information disseminates from a source (leader) to a network of followers. Two types of leader-following graphs will be investigated, namely (i) graphs having a leader connected to an undirected graph, and (ii) directed acyclic graphs. In each case, we derive sufficient conditions for the followers to reach a consensus with the leader's states. Due to the applicability of matrix-weighted consensus algorithms in opinion dynamics processes, we then interpret the results in the context of social behaviors.

The rest of this paper is organized as follows. Section II briefly recalls some background on a matrix-weighted consensus algorithm and formulates the problem. In Section III, we present the theoretical analysis of the proposed consensus protocol under two types of leader-following graphs. In addition, we discuss and interpret these results in the context of social networks carrying out opinion dynamics processes. Section IV contains some simulations to validate the mathematical analysis. Finally, we summarize the paper and discuss further research directions in Section V. 


\section{Preliminaries and Problem Formulation}

Consider a system of $n$ agents (followers) and a single leader. The dynamics of each agent is given by

$$
\dot{\mathbf{x}}_{i}=\mathbf{u}_{i}, \quad \forall i=1, \ldots, n,
$$

where $\mathbf{x}_{i} \in \mathbb{R}^{d}$ and $\mathbf{u}_{i} \in \mathbb{R}^{d}$ are agent $i$, state vector and control input, respectively. The control input of each agent is influenced by its neighbors' states and the matrix weights, but is not influenced by any external input. The leader, labeled as $i=0$, has a constant state $\mathbf{x}_{0} \in \mathbb{R}^{d}$, i.e.,

$$
\dot{\mathbf{x}}_{0}=\mathbf{0} \text {. }
$$

We use a matrix-weighted graph [12] to characterize the information flow in the system. The information flow between the $n$ followers is described by a matrix-weighted graph $\mathcal{G}=(\mathcal{V}, \mathcal{E}, \mathcal{A})$. Here, $\mathcal{V}=\{1, \ldots, n\}$ is the set of vertices representing $n$ followers, $\mathcal{E} \subseteq \mathcal{V} \times \mathcal{V}$ is the set of edges in the graph. An edge of $\mathcal{G}$ between two vertices $i$ and $j, i \neq j$, denoted by $(i, j)$, indicates that information passes from agent $i$ to agent $j$, in at least one state variable. In this paper, we assume there are no self-loops, i.e. $\nexists i:(i, i) \in \mathcal{E}$. The connection between $i$ and $j$ are characterized by a symmetric positive semidefinite matrix $\mathbf{A}_{i j} \in \mathbb{R}^{d \times d}$. For an undirected graph, if $(i, j) \in \mathcal{E}$ then $(j, i) \in \mathcal{E}$ and it is further assumed that $\mathbf{A}_{i j}=\mathbf{A}_{i j}^{T}=\mathbf{A}_{j i} \geq 0$ and $\mathcal{A}=\left\{\mathbf{A}_{j i}:(i, j) \in \mathcal{E}\right\}$ is referred to as the matrix-weight set corresponding to the edge set $\mathcal{E}$. For a directed graph, an edge $(i, j)$ is represented by an arrow from vertex $i$ to vertex $j$, indicating that agent $j$ receives information from agent $i$. If there is an edge $(i, j)$, agent $j$ is called a neighbor of $i$, and we have $\mathbf{A}_{j i}=\mathbf{A}_{j i}^{\top} \geq 0$. Because the graph is directed, $(i, j) \in \mathcal{E}$ does not imply $(j, i) \in \mathcal{E}$, and $\mathbf{A}_{i j}, \mathbf{A}_{j i}$ are not necessarily equal. The set of neighbors of agent $i$ is denoted by $\mathcal{N}_{i}=\{j \in \mathcal{V}:(i, j) \in \mathcal{E}, j \neq i\}$. A (directed) path $\mathcal{P}=i_{1} \ldots i_{k}\left(i_{j} \in \mathcal{V}\right)$ is a sequence of edges $\left(i_{k}, i_{k+1}\right)$ in the graph. A graph is semipositively connected if there is a path between every pair of vertices (note that graphs with scalar edge weights are referred to as being "connected". The semipositivity arises because we have assumed $\mathbf{A}_{i j} \geq 0$.). A directed path $\mathcal{P}$ forms a directed cycle if $i_{1} \equiv i_{k}$. A directed acyclic graph is a directed graph that has no cycles [22].

Corresponding to a graph $\mathcal{G}$, we define the matrixweighted adjacency matrix $\mathbf{A}=\left[\mathbf{A}_{i j}\right] \in \mathbb{R}^{d n \times d n}$ [5]. For any undirected graph $\mathcal{G}$, the matrix-weighted adjacency matrix $\mathbf{A}$ is symmetric. Let $\mathbf{D}_{i}=\sum_{j \in \mathcal{N}_{i}} \mathbf{A}_{i j}$ be the degree matrix of the vertex $i$ and define $\mathbf{D}=\operatorname{blkdiag}\left(\mathbf{D}_{i}\right)$ as the degree matrix of the graph $\mathcal{G}$. The matrix-weighted Laplacian is defined as $\mathbf{L}=\mathbf{D}-\mathbf{A}$. The matrix-weighted consensus protocol defined on graph $\mathcal{G}$ (without a leader) is given by

$$
\dot{\mathbf{x}}_{i}=\sum_{j \in \mathcal{N}_{i}} \mathbf{A}_{i j}\left(\mathbf{x}_{j}-\mathbf{x}_{i}\right), \forall i=1, \ldots, n .
$$

A cluster of a graph $\mathcal{G}$ is a set of vertices $\mathcal{V}^{\prime} \subseteq \mathcal{V}$ such that under the matrix-weighted consensus protocol (3), $\mathbf{x}_{i} \rightarrow \mathbf{x}_{j}$ as $t \rightarrow \infty$ for all $i, j \in \mathcal{V}^{\prime}$. The following result is useful in later analysis:
Lemma 1: [12, Theorem 5] The undirected graph $\mathcal{G}$ has a cluster containing all vertices of $\mathcal{V}$ if and only if the left nullspace of the matrix-weighted Laplacian $\mathbf{L}$ of $\mathcal{G}$ is $\mathcal{N}(\mathbf{L})=\mathcal{R}:=\operatorname{Range}\left(\mathbf{1}_{n} \otimes \mathbf{I}_{d}\right)$.

We study consensus problems with two types of leaderfollowing graphs denoted by $\overline{\mathcal{G}}$. Each graph $\overline{\mathcal{G}}$ has a single leader and is defined as follows. Beginning with the graph $\mathcal{G}$, we add a vertex 0 (representing the leader), a set of directed edges $\mathcal{E}_{0}$ from vertex 0 to some vertices $i \in \mathcal{V}$, and a corresponding set of matrix weights $\mathcal{A}_{0}=\left\{\mathbf{A}_{i 0}, \forall i \in \mathcal{V}\right\}$. The matrix-weight $\mathbf{A}_{i 0}$ is positive semidefinite if agent $i$ has access to the leader's state, i.e. $(0, i) \in \mathcal{E}_{0}$; otherwise, $\mathbf{A}_{i 0}=\mathbf{0}$. The graph $\overline{\mathcal{G}}=\{\overline{\mathcal{V}}, \overline{\mathcal{E}}, \overline{\mathcal{A}}\}$ is directed, with $\overline{\mathcal{V}}=\{0\} \cup \mathcal{V}, \overline{\mathcal{E}}=\mathcal{E}_{0} \cup \mathcal{E}$, and $\overline{\mathcal{A}}=\mathcal{A}_{0} \cup \mathcal{A}$. For problem 1, we assume the follower graph $\mathcal{G}$ is undirected, and for problem 2 , we assume the follower graph $\mathcal{G}$ is directed. Specifically, the following system will be studied for problems 1 and 2:

$$
\begin{aligned}
\dot{\mathbf{x}}_{0} & =\mathbf{0}, \\
\dot{\mathbf{x}}_{i} & =\sum_{j \in \mathcal{N}_{i}} \mathbf{A}_{i j}\left(\mathbf{x}_{j}-\mathbf{x}_{i}\right)+\mathbf{A}_{i 0}\left(\mathbf{x}_{0}-\mathbf{x}_{i}\right),
\end{aligned}
$$

where $i=1, \ldots, n$. The leader-following system (4) is said to achieve leader-follower consensus if, for any initial state $\mathbf{x}_{i}(0) \in \mathbb{R}^{d}, i=1, \ldots, n$, there holds $\lim _{t \rightarrow \infty} \mathbf{x}_{i}(t) \rightarrow \mathbf{x}_{0}$.

\section{A. Opinion Dynamics on Multiple Interdependent Topics}

Here, we give one motivating application of matrixweighted consensus algorithms (among many others). In the context of opinion dynamics, the matrix weight $\mathbf{A}_{i j}$ describes 1) the logical interdependence between multiple topics, and 2) interpersonal relationships that depend on the topics being discussed. The well known consensus algorithm

$$
\dot{\mathbf{x}}_{i}=\sum_{j \in \mathcal{N}_{i}} a_{i j}\left(\mathbf{x}_{j}-\mathbf{x}_{i}\right), \forall i=1, \ldots, n .
$$

where $\mathbf{x}_{i} \in \mathbb{R}^{d}$ is the opinion of individual $i$ on $d$ unrelated topics and $a_{i j}>0$ is a scalar, is also known as the Abelson model [11]. The Abelson model is a continuous-time counterpart to the seminal discrete-time French-DeGroot model for opinion pooling [10], [23].

The consensus algorithm (3) represents discussion on $d$ different topics which are logically interdependent. Consider two topics being simultaneously discussed; 1) your favourite sport and 2) the world's most famous/successful athlete. Clearly an individual's opinion on topic 2 is dependent on his/her opinion on topic 1; a person who enjoys basketball is more likely to believe Michael Jordan is the most famous athlete in contrast to someone who closely follows golf and may choose Tiger Woods. The matrix weight $\mathbf{A}_{i j}$ enables an individual to simultaneously adjust his/her opinions on interdependent issues in order to maintain consistency in his/her set of attitudes and beliefs [13], [14], [24].

In the scalar case (5), $a_{i j}$ can be considered as the strength of the interpersonal relationship between individual $i$ and individual $j$, i.e. how much individual $i$ takes into account the opinion of individual $j$. Notice that for a given individual $v_{i}$ with two different neighbors $v_{j}, v_{k}$, we allow 


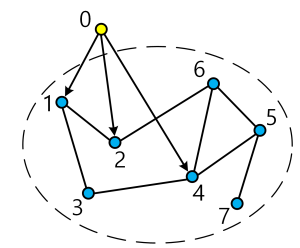

Fig. 1: Leader-follower graph, with undirected follower graph.

$\mathbf{A}_{i j} \neq \mathbf{A}_{i k}$. That is, we allow different matrix weights for different neighbors of $v_{i}$. Suppose that individuals $v_{j}, v_{k}$ are neighbors of individual $v_{i}$, and individual $v_{k}$ is a keen follower of many different sporting codes whereas individual $v_{j}$ seldom watches sports. Then $\mathbf{A}_{i k} \neq \mathbf{A}_{i j}$ enables us to model individual $v_{i}$ 's relationship strengths with $v_{j}, v_{k}$ which differ due to the topics being discussed, i.e. topic-dependent interaction topology.

\section{ANALYSIS AND Discussion}

In this section, we study two common leader-following graphs. We give conditions for reaching a consensus on each type. These conditions are then used to discuss related phenomena in social networks.

\section{A. Undirected Follower Interaction Topology}

Before studying the system (4), we state the following assumptions:

Assumption 1: The graph $\mathcal{G}$ has a cluster containing all vertices in $\mathcal{V}$, or equivalently, $\mathcal{N}(\mathbf{L})=\mathcal{R}$.

Assumption 2: $\sum_{i=1}^{n} \mathbf{A}_{i 0}$ is positive definite.

Denote $\mathbf{z}_{i}=\mathbf{x}_{i}-\mathbf{x}_{0}$, for $i=1, \ldots, n$, and define $\mathbf{z}:=$ $\left[\mathbf{z}_{1}^{T}, \ldots, \mathbf{z}_{n}^{T}\right]^{T} \in \mathbb{R}^{d n}$ as the stacked error vector. Equation (4b) can be rewritten as follows:

$$
\dot{\mathbf{z}}_{i}=\sum_{j \in \mathcal{N}_{i}} \mathbf{A}_{i j}\left(\mathbf{z}_{j}-\mathbf{z}_{i}\right)-\mathbf{A}_{i 0} \mathbf{z}_{i}, i=1, \ldots, n .
$$

In compact form, the dynamics (6), for the entire follower network, can be expressed as

$$
\dot{\mathbf{z}}=-\mathbf{L} \mathbf{z}-\operatorname{blkdiag}\left(\mathbf{A}_{\mathrm{i} 0}\right) \mathbf{z}=-\left(\mathbf{L}+\operatorname{blkdiag}\left(\mathbf{A}_{\mathrm{i} 0}\right)\right) \mathbf{z} .
$$

We state the following lemmas.

Lemma 2: Let $\mathbf{B}_{i}, i=1, \ldots, n$, be $n$ positive semidefinite matrices and $\mathbf{B}=\sum_{i=1}^{n} \mathbf{B}_{i}$. Then, $\mathcal{N}(\mathbf{B})=$ $\bigcap_{i=1}^{n} \mathcal{N}\left(\mathbf{B}_{i}\right)$ and $\mathcal{N}(\mathbf{B}) \subseteq \mathcal{N}\left(\mathbf{B}_{i}\right)$.

Proof: The proof follows by straightforward linear algebra [25].

Lemma 3: Under Assumption 1, the matrix $\mathbf{H}=(\mathbf{L}+$ $\left.\operatorname{blkdiag}\left(\mathbf{A}_{\mathrm{i} 0}\right)\right)$ is positive definite if and only if $\sum_{i=1}^{n} \mathbf{A}_{i 0}$ is positive definite.

Proof: Under Assumption 1, the matrix-weighted Laplacian $\mathbf{L}$ is positive semidefinite and $\mathcal{N}(\mathbf{L})=\operatorname{span}\left\{\mathbf{1}_{\mathrm{n}} \otimes\right.$ $\left.\mathbf{I}_{\mathrm{d}}\right\} \quad\left[12\right.$, Theorem 2]. Since $\operatorname{blkdiag}\left(\mathbf{A}_{\mathrm{i} 0}\right)$ is positive semidefinite, it follows from Lemma 2 that

$$
\mathcal{N}(\mathbf{H}) \subseteq \mathcal{N}(\mathbf{L})
$$

Let $\left\{\mathbf{e}_{k}\right\}_{k=1, \ldots, d}$ be the standard basis of $\mathbb{R}^{d}$, that is, $\mathbf{e}_{k} \in$

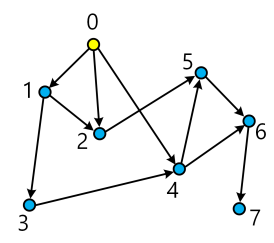

Fig. 2: Leader-follower graph, with directed acyclic follower graph.

$\mathbb{R}^{d}$ is a vector whose $k$-th entry is equal to 1 and other entries are all zero. For any vector $\mathbf{v} \in \mathcal{N}(\mathbf{L})$, we can express $\mathbf{v}$ as a linear combination as follow,

$$
\mathbf{v}=\sum_{k=1}^{d} v_{k}\left(\mathbf{1}_{n} \otimes \mathbf{e}_{k}\right)=\mathbf{1}_{n} \otimes\left(\sum_{i=1}^{d} v_{k} \mathbf{e}_{k}\right),
$$

where $v_{k} \in \mathbb{R}, k=1, \ldots, d$, are scalars. For any $\mathbf{v} \in \mathcal{N}(\mathbf{L})$,

$$
\begin{aligned}
\mathbf{v}^{T} \mathbf{H} \mathbf{v} & =\mathbf{v}^{T}\left(\mathbf{L}+\operatorname{blkdiag}\left(\mathbf{A}_{\mathrm{i} 0}\right)\right) \mathbf{v} \\
& =\left(\mathbf{1}_{n}^{T} \otimes\left(\sum_{k=1}^{d} v_{k} \mathbf{e}_{k}^{T}\right)\right) \operatorname{blk} \operatorname{diag}\left(\mathbf{A}_{\mathrm{i} 0}\right)\left(\mathbf{1}_{\mathrm{n}} \otimes\left(\sum_{\mathrm{k}=1}^{\mathrm{d}} \mathrm{v}_{\mathrm{k}} \mathbf{e}_{\mathrm{k}}\right)\right) \\
& =\sum_{i=1}^{n}\left(\sum_{k=1}^{d} v_{k} \mathbf{e}_{k}^{T}\right) \mathbf{A}_{i 0}\left(\sum_{k=1}^{d} v_{k} \mathbf{e}_{k}\right) \\
& =\left(\sum_{k=1}^{d} v_{k} \mathbf{e}_{k}^{T}\right)\left(\sum_{i=1}^{n} \mathbf{A}_{i 0}\right)\left(\sum_{k=1}^{d} v_{k} \mathbf{e}_{k}\right)
\end{aligned}
$$

Suppose that $\sum_{i=1}^{n} \mathbf{A}_{i 0}$ is positive definite. Then it follows from (10) that $\mathbf{v}^{T} \mathbf{H} \mathbf{v}>0, \forall \mathbf{v} \in \mathcal{N}(\mathbf{L})$. Therefore, (8) implies that $\mathcal{N}(\mathbf{H})=\{\mathbf{0}\}$, i.e., $\mathbf{H}$ is positive definite.

On the other hand, if $\sum_{i=1}^{n} \mathbf{A}_{i 0}$ is positive semidefinite but not positive definite, then we can always choose $v_{k}, k=$ $1, \ldots, d$, such that $\mathbf{v}^{\prime}=\sum_{k=1}^{d} v_{k} \mathbf{e}_{k}^{T} \neq \mathbf{0}$ is an eigenvector of $\sum_{i=1}^{n} \mathbf{A}_{i 0}$ corresponding to a zero eigenvalue. Also, $\mathbf{v}=$ $\mathbf{1}_{d} \otimes \mathbf{v}^{\prime}$ is an eigenvector of $\mathbf{H}$ corresponding to a zero eigenvalue. Thus, $\mathbf{H}$ is positive semidefinite, but singular, in this case.

We can now state the main result for the undirected follower topology problem in the following theorem.

Theorem 1: Under Assumptions 1 and 2, the leaderfollowing system (4) achieves leader-follower consensus, for all initial conditions $\mathbf{x}_{i} \in \mathbb{R}^{d}, i \in \overline{\mathcal{V}}$, exponentially fast.

Proof: Under Assumptions 1 and 2, it follows from Lemma 3 that $\mathbf{H}=\left(\mathbf{L}+\operatorname{blkdiag}\left(\mathbf{A}_{\mathrm{i} 0}\right)\right)$ is positive definite. Because (7) is a linear system $(\dot{\mathbf{z}}=-\mathbf{H z})$ and $\mathbf{H}$ is positive definite, $\mathbf{z}_{i} \rightarrow \mathbf{0}$, as $t \rightarrow \infty$. It follows from the definition of $\mathbf{z}_{i}$ that the system (4) achieves a leader-follower consensus exponentially fast.

Remark 1: The convergence rate of (7) depends on the smallest eigenvalue of $\mathbf{H}, \lambda_{1}(\mathbf{H})$. Further, it is easy to see that $\lambda_{1}(\mathbf{H}) \leq \lambda_{d+1}(\mathbf{L})+\lambda_{1}\left(\sum_{i=1}^{n} \mathbf{A}_{i 0}\right)$.

Theorem 1 implies that clustering behavior happens when either the graph $\mathcal{G}$ does not have a spanning cluster or the communication from the leader to the follower agents in $\mathcal{G}$ is incomplete, i.e., $\sum_{i=1}^{n} \mathbf{A}_{i 0}$ is not positive definite. 


\section{B. Directed Follower Interaction Topology}

In this scenario, we state the following assumption on the system (4):

Assumption 3: The matrix $\mathbf{M}_{i}=\sum_{j=0}^{n} \mathbf{A}_{i j}$ is positive definite for all $i=1, \ldots, n$.

Let $\mathbf{z}_{i}=\mathbf{x}_{i}-\mathbf{x}_{0}, i=1, \ldots, n$, and $\mathbf{z}=\left[\mathbf{z}_{1}^{T}, \ldots, \mathbf{z}_{n}^{T}\right]^{T} \in$ $\mathbb{R}^{d n}$. From (1), we can write the $\mathbf{z}-$ dynamics as follows:

$$
\dot{\mathbf{z}}=-\mathbf{H z},
$$

where $\mathbf{H}=\mathbf{L}+\operatorname{blkdiag}\left(\mathbf{A}_{i 0}\right)$ is the matrix given by

$$
\mathbf{H}=\left[\begin{array}{cccc}
\mathbf{A}_{10} & \mathbf{0} & \cdots & \mathbf{0} \\
-\mathbf{A}_{21} & \sum_{l=0}^{1} \mathbf{A}_{2 l} & \cdots & \mathbf{0} \\
\vdots & \vdots & \ddots & \vdots \\
-\mathbf{A}_{n 1} & -\mathbf{A}_{n 2} & \cdots & \sum_{l=0}^{n} \mathbf{A}_{n l}
\end{array}\right]
$$

The following theorem is the main result for the directed follower topology problem:

Theorem 2: Under Assumption 3, the leader-following system (4) achieves leader-follower consensus, for all initial conditions $\mathbf{x}_{i} \in \mathbb{R}^{d}, i \in \overline{\mathcal{V}}$, exponentially fast.

Proof: Under Assumption 3, we have

$$
\begin{aligned}
\operatorname{det}\left(\lambda \mathbf{I}_{d n}-\mathbf{H}\right) & =\prod_{i=1}^{n} \operatorname{det}\left(\lambda \mathbf{I}_{d}-\sum_{l=0}^{n} \mathbf{A}_{i l}\right) \\
& =\prod_{i=1}^{n} \operatorname{det}\left(\lambda \mathbf{I}_{d}-\sum_{l \in \mathcal{N}_{i}}^{n} \mathbf{A}_{i l}\right) .
\end{aligned}
$$

Since $\mathbf{M}_{i}=\sum_{l \in \mathcal{N}_{i}} \mathbf{A}_{i l}, i=1, \ldots, n$ is positive definite, it follows from (12) that all eigenvalues of $\mathbf{H}$ are strictly positive and real, or i.e., $-\mathbf{H}$ is Hurwitz stable. Thus, the origin $\mathbf{z}=\mathbf{0}$ is a globally exponentially stable equilibrium of the system (11). The definition of $\mathbf{z}_{i}$ then implies that leader-follower consensus is achieved.

The following question naturally arises from considering the result in Theorem 2: "What happens if, for one or more $i, \mathbf{M}_{i}$ is positive semidefinite and singular?" The overall system will not achieve a consensus to the leader's states in this case. However, all follower agents can reach partial agreement on some states.

Since $\mathbf{M}_{i}$ is symmetric, it is always diagonalizable. Let $\mathbf{u}_{k}^{i} \in \mathbb{R}^{d}$, be an eigenvector of $\mathbf{M}_{i}$ corresponding to eigenvalue $\xi_{k}$, where $k=1, \ldots, d$. Then, $\mathbf{v}_{d(i-1)+k}=$ $\left[\begin{array}{lll}\mathbf{0}_{d(i-1)}^{T} & \left(\mathbf{u}_{k}^{i}\right)^{T} & \mathbf{0}_{d(n-i)}^{T}\end{array}\right]^{T} \in \mathbb{R}^{d n}$ is a right eigenvector of $\mathbf{L}$ corresponding to the eigenvalue $\lambda_{d(i-1)+k}=\xi_{k}$ $(i=1, \ldots, n$, and $k=1, \ldots, d)$. Let $\mathbf{r}_{d(i-1)+k}$ be the corresponding left eigenvector of $\mathbf{H}$. Since $\left\{\mathbf{v}_{l}\right\}_{l=1, \ldots, n}$ is a basis consisting of eigenvectors of $\mathbf{H}$, it follows $\mathbf{L}$ is diagonalizable. From linear systems theory [26], solutions of (11) are given by

$$
\begin{aligned}
\mathbf{z}(t) & =\sum_{i=1}^{n} \sum_{k=1}^{d}\left(\mathbf{v}_{d(i-1)+k}^{T} \mathbf{z}(0)\right) \mathbf{r}_{l} e^{-\lambda_{d(i-1)+k} t} \\
& =\sum_{i=1}^{n} \sum_{k=1}^{d}\left(\left(\mathbf{u}_{k}^{i}\right)^{T} \mathbf{z}_{i}(0)\right) \mathbf{r}_{l} e^{-\lambda_{d(i-1)+k} t} .
\end{aligned}
$$

Thus, as $t \rightarrow \infty$, states corresponding to $\lambda_{d(i-1)+k}=0$ are invariant while other states converge to zero. The existence of some zero eigenvalues implies that a consensus is achieved if $\mathbf{z}_{i}(0) \perp \mathcal{N}\left(\mathbf{M}_{i}\right)$, for all $i=1, \ldots, n$, otherwise clustering phenomena occurs.

Clustering has been observed in different variations of the consensus algorithm, but most existing results focus on algorithms concerning scalar edge weights. Negative weights were used to achieve bipartite consensus in [27], and to achieve cluster consensus in [19]. Bounded-confidence algorithms achieved clustering in [28], while individual stubbornness explained clustering phenomenon in [14]. To the authors' knowledge, this paper and the paper [12] are the first to obtain clustering behavior using matrix-weighted consensus algorithms.

\section{Interpreting the results with application to social behav- iors}

In this section, we provide more discussion on the two above problems in the context of behavior in social networks. The leader-follower topologies studied are reflective of structured communities with a clear leader. The leader is responsible for deciding how the whole community reacts to the outside environment which might include outside information. Our first observation is that a decision or piece of information from the leader can disseminate to the community via several information channels, e.g., documents, rules, or announcements, etc. Thus, matrix weights can describe the mixing of dissemination methods in those information channels. Having matrix weights enables us to capture an individual's ability to place different weights on difference information sources. Allowing $\mathbf{A}_{i j}$ to vary between individuals allows for modeling of individual $i$ allocating different trusts for different neighbor $j$.

The undirected follower topology models a general social network. The leader sends pieces of information to a subset $S$ of its followers ( $i \in S$ implies $\mathbf{A}_{i 0}$ is positive semidefinite). In such a social structure, people can exchange information with friends, neighbors, and thus eventually the information from the leader passes to the whole network. The result in Section III-A suggests that the followers can reach a consensus to the leader's states if and only if (i) the network is spanned by a cluster (the community is well organized); and (ii) $\sum_{i \in S} \mathbf{A}_{i 0}$ is positive definite (the aggregated information the leader sent is complete). Information disseminates as fast as the convergence rate of the undirected network.

The second topology is more reflective of a rigid hierarchical society, e.g. a military chain of command. Each follower $i(i=1, \ldots, n)$ has at least one parent individual (an immediate leader). In turn, $i$ may be the immediate leader 
of several other followers. Information can only pass in one direction, from leaders to followers. In this structure, the speed of information spreading to an agent depends on how close it is to the leader. For an agent to reach a consensus with its leader's state, it is required that the connection to its leader, characterized by $\mathbf{M}_{i}=\sum_{j \in \mathcal{N}_{i}} \mathbf{A}_{j i}$, be positive definite. Since communication is unidirectional, in general, this type of network has faster response and requires less communication cost. However, when an agent $i$ does not have enough communication from its leader, or i.e., $\mathbf{M}_{i}$ is only positive semidefinite, then $i$ may not consent with its leader on some states. Consequently, agent $i$ 's followers will also be affected by this misconnection. Thus, the top-down structure is less robust against failure/corruption in the network.

\section{Simulations}

In this section, we provide numerical simulations to illustrate the analysis in Section III. Each simulation consists of two setups, one corresponding to a case where the system reaches a consensus, and the other to the case where clustering behavior is present.

\section{A. Simulation 1: Undirected follower graph}

Consider a system consisting of eight agents in $\mathbb{R}^{2}$ with the interconnection graph as depicted in Fig. 1. The leader states are given as $\mathbf{x}_{0}=[0,2]^{T}$. The initial conditions of the follower agents in the simulation are arbitrarily chosen.

Simulation 1a: In this simulation, the set of matrix weights are given as follows: $\mathbf{A}_{10}=\left[\begin{array}{ll}1 & 0 \\ 0 & 0\end{array}\right], \mathbf{A}_{20}=2 \mathbf{I}_{2}$, $\mathbf{A}_{40}=\left[\begin{array}{ll}0 & 0 \\ 0 & 1\end{array}\right], \mathbf{A}_{13}=\left[\begin{array}{cc}2 & -1 \\ -1 & 2\end{array}\right], \mathbf{A}_{25}=\left[\begin{array}{cc}0.45 & 0.6 \\ 0.6 & 0.8\end{array}\right], \mathbf{A}_{45}=$ $\left[\begin{array}{ll}1 & 1 \\ 1 & 1\end{array}\right], \mathbf{A}_{46}=\left[\begin{array}{ll}9 & 3 \\ 3 & 1\end{array}\right], \mathbf{A}_{56}=\left[\begin{array}{ll}4 & 6 \\ 6 & 9\end{array}\right]$, and $\mathbf{A}_{12}=\mathbf{A}_{34}=$ $\mathbf{A}_{67}=\mathbf{I}_{2}$. It is easy to verify that $\left(\mathbf{A}_{10}+\mathbf{A}_{20}+\mathbf{A}_{40}\right)$ is positive definite. Further, the matrix weights $\mathbf{A}_{12}, \mathbf{A}_{13}, \mathbf{A}_{34}$, and $\mathbf{A}_{67}$ are positive definite, while $\mathbf{A}_{45}, \mathbf{A}_{46}, \mathbf{A}_{25}, \mathbf{A}_{56}$ are positive semidefinite. The fact that the graph $\mathcal{G}$ is spanned by a cluster (Assumption 1) can be verified either by computing the rank of $\mathbf{L}$ as in Lemma 1 , or by using [12, Corollaries 4 and 5].

Simulation results are shown in Fig. 3. It can be observed that all agents asymptotically reach a consensus. The consensus value is precisely the leader's states.

Simulation 1b: In this simulation, we remove the edge $(5,6)$ from $\mathcal{G}$ and keep other edges and their matrix weights unchanged. According to [12, Theorem 3], the graph $\mathcal{G}$ has two clusters: the first cluster contains agents $1, \ldots, 5$, and the other cluster contains agents 6 and 7 .

Simulation results in this case are shown in Fig. 4. Figure 4 shows that agents $1, \ldots, 5$ asymptotically reach the leader's states while agents 6 and 7 reach a consensus on values different from the leader's states. Thus, the simulation result is consistent with the analysis in III-A.

\section{B. Simulation 2: Directed acyclic follower graph}

Consider a system consisting of eight agents in a twodimensional space with the interconnection graph as depicted in Figure 2. The leader states are again given as $\mathbf{x}_{0}=[0,2]^{T}$.

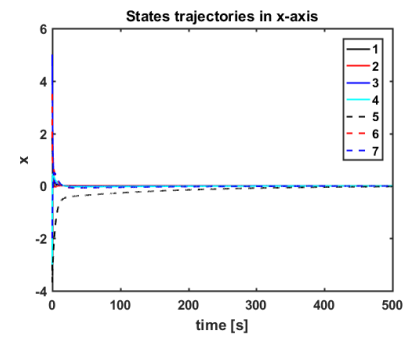

(a) The $x$-axis dynamics.

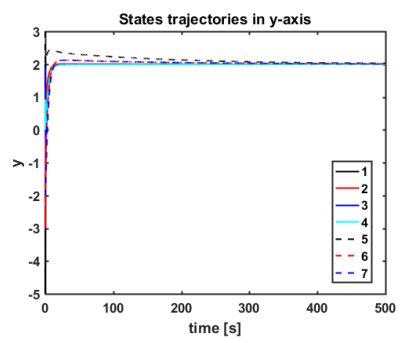

(b) The $y$-axis dynamics.
Fig. 3: Simulation 1a: The agents reach a consensus with the leader's states.

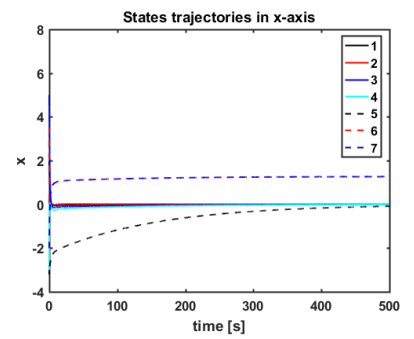

(a) The $x$-axis dynamics.

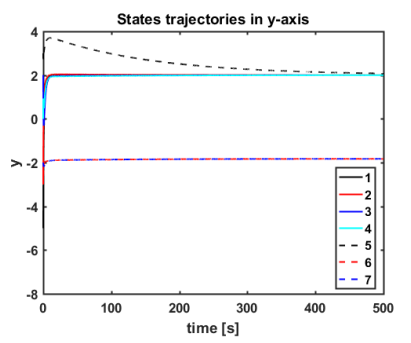

(b) The $y$-axis dynamics.
Fig. 4: Simulation 1b: Agents $1, \ldots, 5$ reach a consensus with the leader while agents 6 and 7 reach a consensus to values different from the leader's states.

Simulation 2a: In this simulation, the set of matrix weights are given as follows: $\mathbf{A}_{10}=\mathbf{A}_{34}=\mathbf{A}_{67}=\mathbf{I}_{2}, \mathbf{A}_{20}=$ $\left[\begin{array}{ll}1 & 0 \\ 0 & 0\end{array}\right], \mathbf{A}_{12}=\left[\begin{array}{ll}0 & 0 \\ 0 & 2\end{array}\right] \quad \mathbf{A}_{40}=\left[\begin{array}{ll}0 & 0 \\ 0 & 1\end{array}\right], \mathbf{A}_{13}=\left[\begin{array}{cc}2 & -1 \\ -1 & 2\end{array}\right]$, $\mathbf{A}_{25}=\left[\begin{array}{cc}0.45 & 0.6 \\ 0.6 & 0.8\end{array}\right], \mathbf{A}_{45}=\left[\begin{array}{ll}1 & 1 \\ 1 & 1\end{array}\right], \mathbf{A}_{46}=\left[\begin{array}{ll}9 & 3 \\ 3 & 1\end{array}\right]$, and $\mathbf{A}_{56}=$ $\left[\begin{array}{ll}4 & 6 \\ 6 & 9\end{array}\right]$. Note that $\mathbf{A}_{20}, \mathbf{A}_{12},, \mathbf{A}_{25}, \mathbf{A}_{40}, \mathbf{A}_{45}, \mathbf{A}_{46}$, and $\mathbf{A}_{56}$ are positive semidefinite. Also, it is easy to verify that Assumption 3 is satisfied.

Simulation results are shown in Fig. 5. All agents asymptotically reach a consensus with the leader as expected. Note that the speed of reaching consensus in this simulation is faster than in Simulation 1a, which is consistent with the discussion in III-C.

Simulation 2b: In this simulation, we remove the edge $(3,4)$ from $\mathcal{G}$ and keep other edges unchanged. After removing $(3,4)$, the rank condition on Assumption 3 is only satisfied for agents 1,2 , and 3 .

The results are depicted in Fig. 6. It can be observed from Figs. $6 \mathrm{a}$ and $6 \mathrm{~b}$ that four clusters appear in this case. Three agents 1,2, and 3 reach a consensus to the leader's states. Agents 4, 5 converge to a different cluster. The last cluster contains agents 6 and 7. The states of agents 6 and 7 converge to a common value due to the fact that the matrix weight $\mathbf{A}_{67}$ is positive definite. Simulation results in this case are also consistent with the analysis in III-B.

\section{CONCLUSIONS}

In this paper, we studied a matrix-weighted consensus algorithm on undirected and directed leader-following interac- 


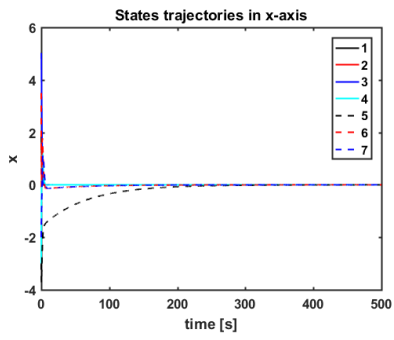

(a) The $x$-axis dynamics.

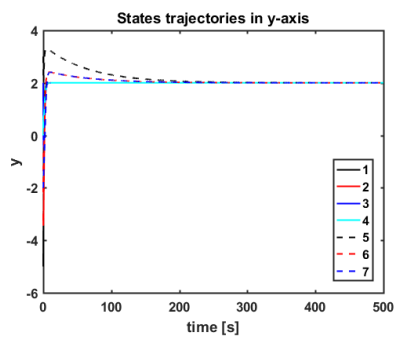

(b) The $y$-axis dynamics.
Fig. 5: Simulation 2a: The agents reach a consensus with the leader's states.

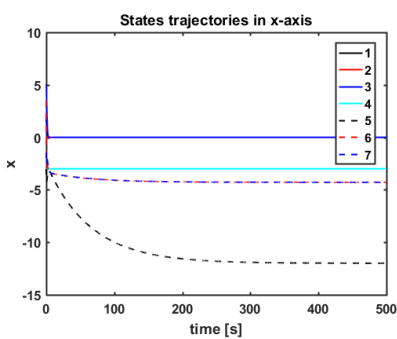

(a) The $x$-axis dynamics.

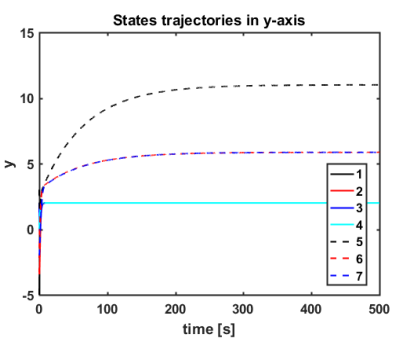

(b) The $y$-axis dynamics.
Fig. 6: Simulation 2b: Agents 1, 2, 3 reach a consensus with the leader's states while other agents reach a consensus on some different values.

tion graphs. Conditions for reaching consensus or clustering were investigated in both cases. Furthermore, we interpreted algorithms and results in the context of social networks. Finally, simulations were given to validate the analytical results.

For further studies, it is of interest to find conditions for reaching a consensus in leader-following graphs with fixed or dynamic multiple leaders. Study of the matrix-weighted consensus protocol under switching leader-following graphs, or in the presence of communication delays/random perturbations are also possible extensions. These extensions may lead to a deeper understanding on how information is disseminated in social networks.

\section{ACKNOWLEDGEMENT}

The work of M. Ye and B.D.O. Anderson was supported by the Australian Research Council (ARC) under DP-160104500, and by Data61-CSIRO. M. Ye was supported by an Australian Government Research Training Program (RTP) Scholarship. The work of M.H. Trinh and H.-S. Ahn was supported by GIST Research Institute (GRI) and by the National Research Foundation (NRF) of Korea under the grant NRF-2017R1A2B3007034.

\section{REFERENCES}

[1] W. Ren, R. W. Beard, and T. W. McLain, "Coordination variables and consensus building in multiple vehicle systems," in Cooperative Control. Springer, 2005, pp. 171-188.

[2] R. Olfati-Saber, J. A. Fax, and R. M. Murray, "Consensus and cooperation in networked multi-agent systems," Proceedings of the IEEE, vol. 95, no. 1, pp. 215-233, 2007.
[3] Y. Cao, W. Yu, W. Ren, and G. Chen, "An Overview of Recent Progress in the Study of Distributed Multi-Agent Coordination," IEEE Transactions on Industrial Informatics, vol. 9, no. 1, pp. 427-438, Feb 2013.

[4] J. A. Fax and R. M. Murray, "Information flow and cooperative control of vehicle formations," IEEE Transactions on Automatic Control, vol. 49, no. 9, pp. 1465-1476, 2004.

[5] S. E. Tuna, "Synchronization under matrix-weighted Laplacian," Automatica, vol. 73, pp. 76-81, 2016.

[6] B.-Y. Kim, K.-K. Oh, K. L. Moore, and H.-S. Ahn, "Distributed coordination and control of multiple photovoltaic generators for power distribution in a microgrid," Automatica, vol. 73, pp. 193-199, 2016.

[7] W. Ren, R. W. Beard et al., "Consensus seeking in multiagent systems under dynamically changing interaction topologies," IEEE Transactions on automatic control, vol. 50, no. 5, pp. 655-661, 2005.

[8] N. E. Friedkin, "The Problem of Social Control and Coordination of Complex Systems in Sociology: A Look at the Community Cleavage Problem," IEEE Control Systems Magazine, vol. 35, no. 3, pp. 40-51, 2015.

[9] G. Shi and K. H. Johansson, "The Role of Persistent Graphs in the Agreement Seeking of Social Networks," IEEE Journal on Selected Areas in Communications, vol. 31, no. 9, pp. 595-606, September 2013.

[10] M. H. Degroot, "Reaching a consensus," Journal of the American Statistical Association, vol. 69, no. 345, pp. 118-121, 1974.

[11] R. P. Abelson, "Mathematical models of the distribution of attitudes under controversy," Contributions to mathematical psychology, vol. 14, pp. 1-160, 1964.

[12] M. H. Trinh and H.-S. Ahn, "Theory and applications of matrixweighted consensus," https://arxiv.org/abs/1703.00129.

[13] S. E. Parsegov, A. V. Proskurnikov, R. Tempo, and N. E. Friedkin, "Novel Multidimensional Models of Opinion Dynamics in Social Networks," IEEE Transactions on Automatic Control, to appear.

[14] N. E. Friedkin, A. V. Proskurnikov, R. Tempo, and S. E. Parsegov, "Network science on belief system dynamics under logic constraints," Science, vol. 354, no. 6310, pp. 321-326, 2016.

[15] S. E. Tuna, "Conditions for synchronizability in arrays of coupled linear systems," IEEE Transactions on Automatic Control, vol. 54, no. 10 , pp. $2416-2420,2009$.

[16] S. Zhao and D. Zelazo, "Bearing-based formation stabilization with directed interaction topologies," in Proc. of the 54th IEEE Conference on Decision and Control (CDC), 2015, pp. 6115 - 6120.

[17] _ _ "Localizability and distributed protocols for bearing-based network localization in arbitrary dimensions," Automatica, vol. 69, pp. 334-341, 2016.

[18] W. Ni and D. Cheng, "Leader-following consensus of multi-agent systems under fixed and switching topologies," Systems and Control Letters, vol. 59, pp. 209-217, 2010.

[19] J. Qin, C. Yu, and B. D. O. Anderson, "On leaderless and leaderfollowing consensus for interacting clusters of second-order multiagent systems," Automatica, vol. 74, pp. 214-221, 2016.

[20] J. Hu and Y. Hong, "Leader-following coordination of multi-agent systems with coupling time delays," Physica A: Statistical Mechanics and its Applications, vol. 374, no. 2, pp. 853-863, 2007.

[21] Y. Cao, W. Ren, and M. Egerstedt, "Distributed containment control with multiple stationary or dynamic leaders in fixed and switching directed networks," Automatica, vol. 48, no. 8, pp. 1586-1597, 2012.

[22] C. Godsil and G. Royle, Algebraic graph theory. Springer, 2001.

[23] J. R. P. French Jr., "A formal theory of social power," Psychological Review, vol. 63, no. 3, pp. 181-194, 1956.

[24] B. Gawronski and F. Strack, Cognitive Consistency: A Fundamental Principle in Social Cognition. Guilford press, 2012.

[25] R. Horn and C. Johnson, Matrix Analysis. Cambridge University Press, 1990.

[26] P. Antsaklis and A. N. Michel, Linear Systems. Springer Science \& Business Media, 2006.

[27] C. Altafini, "Consensus problems on networks with antagonistic interactions," IEEE Transactions on Automatic Control, vol. 58, no. 4, pp. 935-946, 2013.

[28] R. Hegselmann and U. Krause, "Opinion dynamics and bounded confidence models, analysis, and simulation," Journal of Artificial Societies and Social Simulation, vol. 5, no. 3, 2002. 